\title{
Optimal Research of Equipment Maintenance Interval Based on Monte-Carlo Simulation
}

\author{
Xianghui Meng ${ }^{1, a}$, Shaohua Wang ${ }^{2, b}$ \\ ${ }^{1}$ Troop 91872, Beijing 102442, China, Beijing \\ ${ }^{2}$ Department of Technology Support Engineering Academy of Armored Force Engineering Beijing, \\ 100072, China \\ aemail: mxh0823@163.com, bemail: aafe77330@@163.com
}

Keywords: Monte-Carlo; Failure Rate; Maintenance Interval

\begin{abstract}
The optimization of maintenance interval of new equipment by simulation is brought forward according to related maintenance requirement and reality. According to the features of equipment, the basic hypothesis that failure rate increase linearly, deteriorate coefficient is opted to adjust failure rate in order to illustrate the effect of negative proactive maintenance or break-down maintenance, then the optimization model is constructed based on Monte-Carlo simulation. The applicability of the model is validated by optimization of maintenance interval about a case aiming at maximizing the readiness.
\end{abstract}

\section{Introduction}

As the new equipment are intensively deployed and put into use, optimization of maintenance support decisions for equipment has become a prominent practical problem. Time-based PM is still the main tasks among the maintenance measures. However, the reasonableness and maintenance intervals of equipment will have a huge impact on readiness and maintenance costs. Normally, maintenance intervals are optimized based on data optimization, artificial intelligence, or simulation-based optimization methods. In the context of insufficient data, simulation-based optimization methods are proved well fitted with deterioration state and failure process, which makes it feasible in the optimization analysis for maintenance intervals of new equipment.

In order to improve the relevance of equipment maintenance, it' $s$ necessary to consider the impact of imperfect repair on deteriorate process of the equipment or the components. Especially during the training or combat, more frequent $\mathrm{CM}$ will not be able to repair the equipment "as good as new ", ignorance of this factors probably will lead to relative long maintenance intervals, however, the rate of CM will increase correspondingly. Therefore, simulation methods are opted in maintenance interval optimization for new equipment.

\section{Acronym}

$\begin{array}{ll}\text { PM } & \text { Preventive Maintenance } \\ \text { CM } & \text { Corrective Maintenance } \\ \text { MCS } & \text { Monte Carlo Simulation }\end{array}$

\section{Monte Carlo Simulation Methods}

Monte Carlo Simulation (MCS) simulates the real function and development regulation of the system by apply random generate process, so as to achieve the purpose of reveal fundamental system function rules. MCS has simple program structure, flexible simulation process, which makes it suitable for solving the multi-dimensional problems ${ }^{[1]}$. So MCS is selected as simulation method for degradation process of equipment.

The basic principle of the MCS is[1]:

$$
Y=f\left(X_{1}, X_{2}, \cdots, X_{n}\right)
$$


Where $X_{1}, X_{2}, \cdots X_{n}$ are random variables, $Y$ is the dependent variable, $f$ is the function formula. However, $f\left(X_{1}, X_{2}, \cdots X_{n}\right)$ is quite complicated in most practical issues, sometimes even absolutely unknown. So it's hard to calculate the probability distribution of $Y$ and its mathematical characteristic with analytic method. MCS is able to directly or indirectly sampled values of each set variables $\left(X_{1 i}, X_{2 i}, \cdots X_{n i}\right)$ with a random number generator, then calculate the value $Y_{i}$ according to equation (1). A set of sampling data of $Y$ is obtained by repeating the sampling process. The estimated probability distribution function of $Y$ and its mathematical characteristics will get close to the actual situation as the increase of the simulation times. The accuracy of $Y$ can be presented by the standard error of the estimated value.

\section{Optimal Modeling of Maintenance Interval of Equipment Based on MCS}

Time-based Maintenance Interval Model. The time-based maintenance interval model is presented in Figure 1. As showed in the figure, $t$ is the maintenance interval, $T_{p}$ is the average PM time, $T_{c}$ is the average CM time, $T_{1}$ is expect time to failure after last preventive maintenance, $T_{2}$ is expect time to $\mathrm{PM}$ after last $\mathrm{CM}, t=T_{1}+T_{2}{ }^{[2,3]}$.

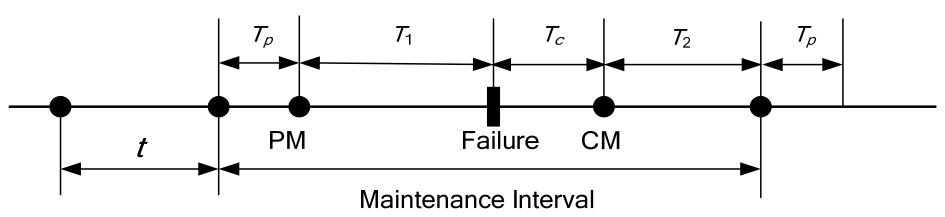

Fig.1 Time-based maintenance interval model

Equipment are complicated system integrated with varied kinds of parts, the failure law of equipment mostly comply with the typical bathtub curve, equipment life stage is divided into infant mortality, normal useful life and wear-out stage. The purpose to perform maintenance is to extend the duration in the normal useful life of the equipment to some extent, so as to achieve the purpose of extending useful life.

The failure modes of equipment are varied corresponding to its structural complexity. As illustrated by Dire Nick, repairable complicated system normally have constant failure rate. However, the reality is often revealed to increase along with the time when mechanical failure is the main failure mode. Based on the analysis, the failure rate $\lambda$ is assumed to rise linearly with the running time during the normal useful life:

$$
\lambda=g\left(t_{h}-t_{h 0}\right)+\lambda_{0}
$$

In the equation, $t_{\mathrm{h}}$ is used to denote the current running time, $t_{\mathrm{h} 0}$ the finish time of the last benign $\mathrm{PM}, \lambda_{0}$ is used to denote the basic failure rate during the normal useful life, $g$ the increment of $\lambda$ per unit of time.

Maintenance will lead to different consequences due to maintenance causes, maintenance techniques, the ability of personnel and maintenance resources. According to the consequences, maintenance is divided into benign PM, poor PM and CM. The failure rate is modified correspondingly to reflect the effect of different kind of maintenance. It's assumed that benign PM can be "as good as before", so the failure rate and maintenance intervals remain unchanged after benign PM:

$$
\lambda_{0}=\lambda_{n}, \tau_{0}=\tau_{n}
$$

$\lambda_{o}$ and $\tau_{o}$ are used to denote the failure rate and maintenance interval before maintenance, $\tau_{n}$ are used to denote the failure rate and maintenance interval after maintenance. CM and poor PM are both imperfect which lead to the increment of failure rate. The function is:

$$
\lambda_{n}=\lambda_{0}(1+\omega)
$$

Maintenance interval simultaneously will be shorted as showed in Eq. (5):

$$
\tau_{n}=\tau_{0} /(1+\omega)
$$

Where $\omega$ is deteriorate coefficient of failure rate which is a positive value. The failure rate and maintenance interval are both modified by $\omega$, corresponding process is showed in Figure 2 , where 
PM and CM processes are both showed.

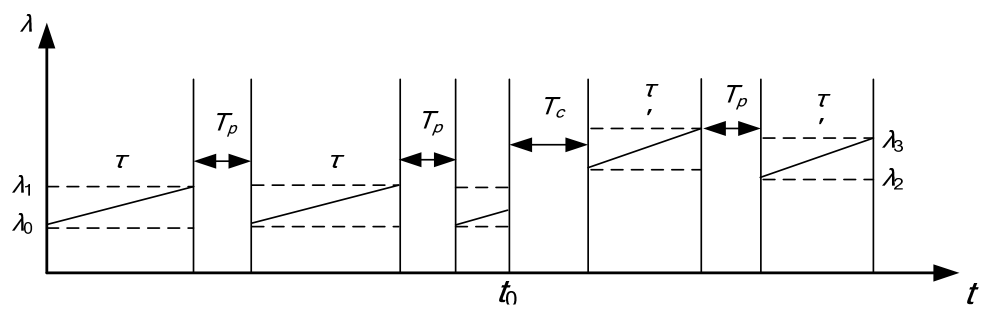

Fig.2 The change process of failure rate

In the figure, $T_{p}$ is used to denote preventive time; $T_{c}$ is used to denote corrective maintenance time. In the first phase, no failure occurred before the PM, the failure rate accumulate from $\lambda_{0}$ to $\lambda_{1}$, the second phase is similar to the first phase, in the third phase the equipment failed at $t_{0}, \mathrm{CM}$ is performed which leads to instantaneous increase to $\lambda_{2}$ after the maintenance, the maintenance interval is reduced from $\tau$ to $\tau$.

As a result, the flat bottom part of classic bathtub curve is replaced by a jagged fold line; the influence on the equipment is illustrated by the increase rate of failure rate. However, the overall increment of failure rate is not obvious so the deteriorate process still can be taken as normal useful life.

Objective Function of the model. The best maintenance interval is determined according to correlated decision-making target. General optimize principles determined based on the characteristic of equipment and corresponding missions are:

(1) Maximization of operational readiness;

(2) Minimization of loss which might be integration of PM expenditure, failure loss and maintenance expenditure, etc.

The readiness of equipment is selected as the optimization target to determine the best maintenance interval. Achieved availability is opted as the decision variable to be maximized, the objective function is:

$\operatorname{MaxA}_{a}=1-\left(M_{c t}+M_{p t}\right) / T_{\text {age }}$

$A_{a}$ is used to denote achieved availability, $M_{c t}$ the accumulated CM time, $M_{p t}$ the accumulated PM time and $T_{\text {age }}$ the equipment lifespan.

Constraints of the model are:

(1) Sampling time $t_{i} \leq T_{\text {age }}$, which is able to make sure maintenance performed during the available lifespan;

(2) $t_{i}-t_{i-1}<\tau_{t i}$, which means that sample interval should be shorter than PM interval.

Simulation of the Model. 1) Simulate process of the model. According to the predefined terms, the state of equipment could be normal, PM downtime and CM downtime which is denoted respectively with $S_{1}, S_{2}$ and $S_{3}$. The state transition process is ${ }^{[4,5]}$ :

(1) If the simulate time reach current interval $\tau_{t}$, the state is changed from $S_{1}$ to $S_{2}$, simulate time push on by $T_{p}$, the state change back to $S_{1}$;

(2) If the simulate time haven't reach current interval $\tau_{t}$, generate a random number uniformly distributed at [0,1], if $r<\lambda\left(t_{h}\right)$, the state is changed from $S_{1}$ to $S_{3}$, simulate time push on by $T_{c}$, the state change back to $S_{1}$, else the state stay unchanged, simulate time push on by one step $T_{\text {step }}$.

2) The calculation of the objective function. PM time and CM time are both assumed to be constant, which means that maintenance interval is the key factor to determine the achieved availability. The variable $\tau$ is set as the input of the model, corresponding achieved availability is calculated, the $\tau^{*}$ which lead to the maximized achieved availability is the optimized interval.

Feasible range for $\tau$ is set to be $\left[\tau_{\min }, \tau_{\max }\right]$ according to similar experience, the achieved availability is calculated every time $\tau$ increase by $\Delta \tau$, the maximized $A_{a}$ and optimized $\tau^{*}$ can be obtained.

In simulation process, the simulate step is denoted as $T_{\text {step }}$, real time point as $T_{\text {now }}$, total PM time $S T_{p}$, total CM time as $S T_{c}$, the lifespan of equipment $T_{a g e}, \lambda$ failure rate, $\lambda_{f}$ poor maintenance threshold. The solving process of the model is presented in Figure 3. 


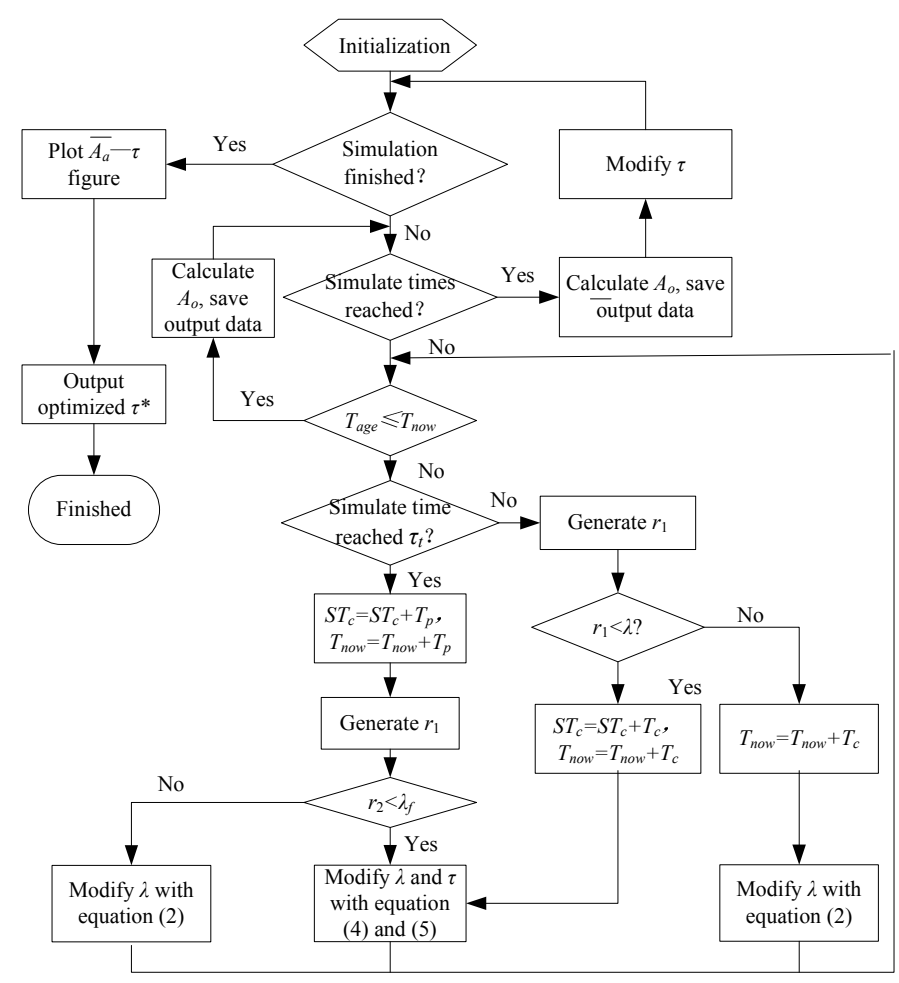

Fig.3 Monte-Carlo simulate process

\section{Case Analysis}

The torque converter system in one equipment is selected as the research object. Corresponding optimization of maintenance intervals is performed. It's easy to recognize that the failure law of the system is in line with the basic assumptions of this paper, so PM is feasible and applicable maintenance work type. MCS is used to optimize its regular maintenance intervals.

The normal failure rate of the system is set to 0.15 according to expert advisement. The designed lifespan is 10 years; the object's finished service time is 1 year. Hypothesis that there are 250 available workdays per year, thus the start time of simulation is set to 250; the stop time correspondingly is set to 2500.Average PM time and CM time is respectively set to 1 day and 2.5 day; poor preventive maintenance rate $\lambda_{f}=0.001$; modify coefficient $\omega=1.002$; increase rate of failure rate $g=0.0001$; simulate step $T_{\text {step }}=10 ;\left[\tau_{\min }, \tau_{\max }\right]$ is assigned as[30,1000].

Run the simulate step according to Figure 3, the threshold of simulation at each interval $\tau$ is set to 100 , calculate $\bar{A}_{o}$ each time the simulation is finished, the $\bar{A}_{o}-\tau$ curve in presented in Figure 4 .

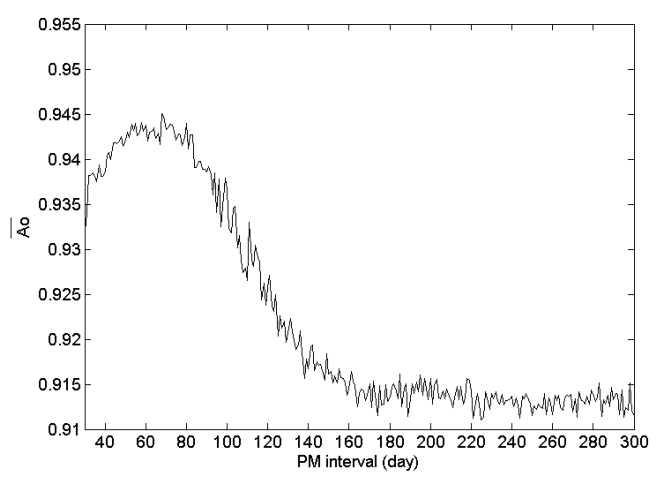

Fig.4 Monte-Carlo simulate output

Theoretically, if PM interval is too small, overall preventive time will be far longer; if PM interval is over large, overall PM time will decrease along with rapid increase in CM time resulted from increased failure times; while reasonable PM interval is able to make trade-off between the 2 kinds of maintenance behaviors. In reality, there exist the unique value comply with the theory. As 
showed in Figure 4, there is obvious change trend of $\bar{A}_{o}$ along with $\tau$. When $\tau=70, \bar{A}_{o}$ is maximized to 0.945 , so the optimized PM interval is set to 70 days. It's necessary to mark that the optimized interval is 70 achievable workday.

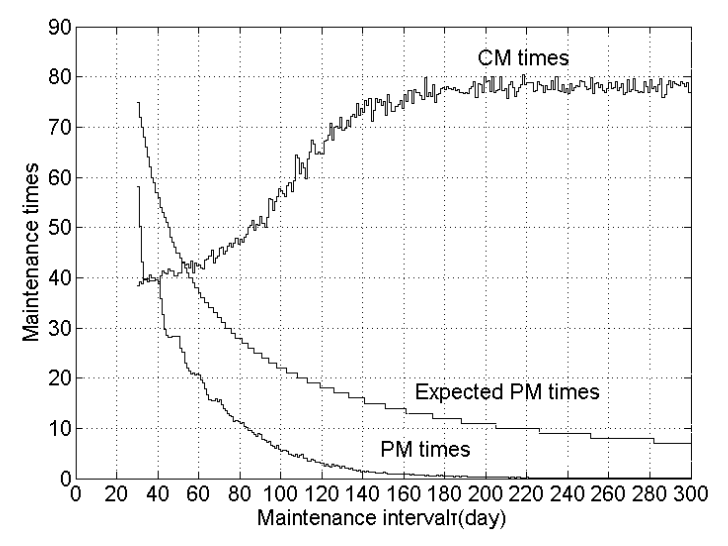

Fig.5 Statistical suspend times resulted from maintenance

The execute times of varied kinds of maintenance is observed at each maintenance interval, as showed in Figure 5. The 3 curve respectively showed CM times, expected PM times and actual PM times, where expected preventive means that no failure occurred before PM. It's easy to find that when $\tau$ is between 30 and 70, CM times almost stay unchanged with a fast decrease of PM times, which means that intensive PM is unnecessary. When $\tau$ reached 200, actual PM times has almost decreased to 0 , while at the same time CM times climb up to 75-80 after a rapid increase phase, in other words PM has become invalid. When $\tau=70$, timely PM is able to limit the probability of failure with acceptable preventive intervention, so it's reasonable to set $\tau^{*}$ as 70 .

\section{Conclusion}

This paper applied MCS into the optimal research of time-based preventive maintenance interval of equipment. MCS is utilized to simulate the change process of failure rate, the occurrence of failure, different kinds of maintenance and related effect based on assumption brought forward in the paper. The model is able to offer valuable information for decision-making in maintenance with data analysis which is easy to understand. The method is suitable for the decision-making and optimization in maintenance of equipment when it's in short of practical running data and failure records.

However, the change law of state is getting more diversified in modern equipment, besides the affection from multiple failure modes; the failure of equipment is also caused by other factors such as manufactory, work load and environment. The model presented in the paper still need to be validated in practice.

\section{References}

[1] Xu Zhongji. Monte-Carlo Simulation Methods[M]. Shanghai Science and Technology Press, 1985.

[2] Fan Yanyan, Jing Denglong. Characteristic of mechanical fault and determination of the best maintenance interval [J]. Aluminum \& Magnesium Communication, 2003 5(3) 56-57.

[3] Marorells, Mun oz A, Serradell VS. Age dependent models for evaluating risks and costs of surveillance and maintenance of components[J]. Reliability Engineering and System Safety, 2000 67(1) 61-73.

[4] Jorn Vatn. Maintenance optimization from a decision theoretical point of view[J]. Reliability Engineering and System Safety, 1997 58(2) 119-126.

[5] Al Dubi. Analytic approach \& Monte-Carlo methods for realistic systems analysis[J]. Mathematics and Computers in Simulation,1998 47(3) 243-269. 